\title{
Large-scale e-mentoring: outcomes and lessons learned
}

\section{Carol Muller}

Carol B. Muller, "Large-scale e-mentoring: outcomes and lessons learned," Proc. SPIE 9663, Eighth International Topical Meeting on Education and Training in Optics and Photonics, 96630Q (6 October 2003); doi: $10.1117 / 12.2208446$

SPIE Event: Eighth International Topical Meeting on Education and Training in Optics and Photonics, 2003, Tucson, Arizona, United States 


\title{
Large-scale e-mentoring: outcomes and lessons learned
}

\author{
Carol B. Muller \\ MentorNet, c/o COE, SJSU, One Washington Square, San Jose, CA 95192-0080 \\ telephone: (408) 924-4070; fax: (408) 924-4069; e-mail: cbmuller@mentornet.net
}

\begin{abstract}
MentorNet (www.MentorNet.net), the e-mentoring network for women in engineering and science, matches undergraduate and graduate students with professionals working in industry for year-long, structured, one-on-one, email-based mentoring relationships, to advance professional development and encourage retention. Collaborating with higher education, industry, government, and professional societies, MentorNet paired nearly 20,000 individuals 1998-2002. (C2003 Optical Society of America OCIS codes: (000.2060) Education
\end{abstract}

MentorNet (www.MentorNet.net), the e-mentoring network for women in engineering and science, is a nonprofit organization designed to further women's progress in scientific and technical fields through a dynamic, technologysupported mentoring program and to advance women and society by developing a diversified, expanded and talented workforce. MentorNet leverages technology to build large-scale impact for women and positive social change, scale which has increased since MentorNet's founding in 1997.

Using a web site and email as basic user-interface, with an underlying database for MentorNet participants and constituents. To match students and mentors, MentorNet has developed a set of sophisticated bi-directional systems using algorithms to optimize strong matches across the pool of thousands of students and mentors. Other systems notify students and mentors by email once a match has been selected for them, provide students and mentors with a series of 16-20 "coaching" messages via email every 1-2 weeks over the course of their mentoring relationship, and internal systems help program managers track current status of mentoring relationships, as well as any issues they arise.

Since 1998, nearly 10,000 undergraduate and graduate women studying engineering and related sciences at more than 100 colleges and universities across the U.S. and in several other nations, have been matched in MentorNet's one-on-one, structured, email-based mentoring relationships with male and female scientific and technical professionals working in industry and government, representing more than 900 different employers. The MentorNet online community also offers E-Forum - a series of topic-based online discussion groups, Bits' $n$ 'Bytes - a monthly newsletter for community members, a resume database for students interested in employment searchable by MentorNet's primary sponsors, and other related resources. In 2002-2003, there were more than 10,000 active members of the community.

MentorNet is committed to ongoing evaluation of programs, for both continuous quality improvement, and measurement of outcomes. Participation in MentorNet's One-on-One program appears to increase student confidence, an important finding since several studies have highlighted confidence as one key gender difference in attrition rates of men and women from the study of engineering. Participation in MentorNet's One-on-One program also appears to contribute to increased interest in industry careers. It is apparent that students value their MentorNet experience, and feel it was worthwhile; based on data collected in 2002, 94\% of students say they would recommend MentorNet to a friend. PhD students, upper division and undergraduates, and African-American and Hispanic students, find MentorNet particularly satisfying.

There are also benefits for mentors, just beginning to be explored. Mentors most frequently cite being able to help someone else as the most valuable aspect of their experience. Other benefits for some mentors include renewed commitment to their field, and improved supervisory, management, and communications skills. Mentors spend an average of just 13.2 minutes per week participating in MentorNet. Mentors also express considerable satisfaction with their experiences with MentorNet; in $2002,96 \%$ of mentors indicated they would recommend MentorNet to a colleague.

Challenges ahead include program sustainability. Built initially with start-up grants providing resources, MentorNet's long term sustainability depends upon increasing the numbers of participating colleges and universities, corporations, and government sites. The current economic climate has challenged this growth plan, and a combination of expense reductions in part involving technology development to reduce staffing, increased marketing to expand scale, and board leadership in driving fund raising will be required to meet future goals.

Eighth International Topical Meeting on Education and Training in Optics and Photonics,

edited by Barry L. Shoop, Grover Swartzlander Jr., Proc. of SPIE Vol. 9663, 96630Q

(C) 2003 SPIE, OSA, ICO · doi: 10.1117/12.2208446 